\title{
Correction to: Geodynamics and Earth Tides Observations from Global to Micro Scale
}

\section{Carla Braitenberg and Giuliana Rossi}

\author{
Correction to: \\ C. Braitenberg, G. Rossi (eds.), Geodynamics and Earth Tides Observations from Global \\ to Micro Scale, Pageoph Topical Volumes, https://doi.org/10.1007/978-3-319-96277-1
}

The below mentioned chapters were published as Open access with incorrect Copyright holder name as "CC Springer Nature Switzerland AG". This has now been updated as "C) The Author(s)" in these chapters. Accordingly, the Cover and the FrontMatter have been updated to reflect these changes.

1. Interferometric Water Level Tilt Meter Development in Finland and Comparison with Combined Earth Tide and Ocean Loading Models

2. Multichannel Singular Spectrum Analysis in the Estimates of Common Environmental Effects Affecting GPS Observations

3. A Filtering of Incomplete GNSS Position Time Series with Probabilistic Principal Component Analysis

The updated online versions of the chapters can be found at https://doi.org/10.1007/978-3-319-96277-1

https://doi.org/10.1007/978-3-319-96277-1_7

https://doi.org/10.1007/978-3-319-96277-1_17

https://doi.org/10.1007/978-3-319-96277-1_19 